\title{
Die Rolle gemeinsamer Urteile für das freie Handeln
}

\author{
PIRMIN STEKELER-WEITHOFER, LEIPZIG
}

Wenn Wörter ihre Bedeutung verlieren, werden die Menschen ihre Freiheit verlieren.

F.A. von Hayek, frei nach Konfuzius ${ }^{1}$

\section{Von Theorien des Spracherwerbs zur Analyse von sozialen Normen}

\subsection{Behavioristische und regulistische Sprachlerntheorien}

Ausgangspunkt der folgenden Überlegungen sind die Entwicklungen, welche in den letzten Jahrzehnten die Philosophie der Sprache und des Geistes genommen haben. Denn spätestens seit Quine, Chomsky und Davidson hat sich in der Sprachphilosophie die Fragestellung verschoben. Zunächst nämlich stand die Frage nach einer rekonstruktiven Bestimmung der Bedingungen hinreichend klarer und deutlicher bzw. wissenschaftlich respektabler Ausdrucksformen in Zentrum. Sie prägt die Philosophie des 20. Jahrhunderts vom Wiener Kreis (Schlick, Carnap) bis zur konstruktiven Wissenschaftsphilosophie der Erlanger Schule Paul Lorenzens, die trotz aller ihrer Mängel in ihren Leistungen im allgemeinen leider bis heute unterschätzt wird. Die neue Frage lautet dagegen, wie die Sprachfähigkeit der Menschen zu erklären sei, wie sie eine Sprache gebrauchen und zu verstehen lernen, und zwar so, dass sie aufgrund endlich vieler Beispielsfälle potentiell unendlich viele Äußerungen und Sätze verstehen, bzw. auf angemessene Weise gebrauchen können. Tiere können das, wie wir wissen, nicht.

Es handelt sich bei dieser Verschiebung der Fragestellung, wie Chomsky klar sieht, um eine kognitionstheoretische Wende weg von einer vorher

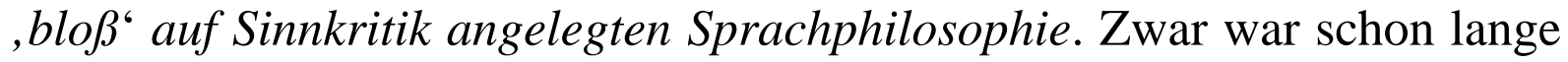

\footnotetext{
${ }^{1}$ Vgl. von Hayek 1996, 115.
} 
vorher klar, dass ein angemessenes Verständnis geistigen Lebens wesentlich einer Klärung der Formen sprachlich gestützten gemeinsamen Handelns bedarf. Es ist eine uralte Einsicht, dass im wesentlichen die Sprachfähigkeit den Menschen zum Menschen macht. Wir sollten bei ihr bleiben. In der neuen Kognitionstheorie möchte man nun aber die sprachlichen und damit die geistigen Fähigkeiten auf natürliche Weise, sprich: evolutionsund lerntheoretisch erklären. Diese Erklärungen sollen, und eben das ist das Erbe des Wiener Kreis, eingebettet werden in den Denkrahmen einer Einheitswissenschaft bzw. in einen zugehörigen Naturalismus. Diesen hat man sich durchaus als eine Fortsetzung des wissenschaftlichen Monismus der 1910er Jahre vorzustellen. Eine vermeintlich oder wirklich bis in die Normalsprache des Alltags und ihre folk-psychology wirkmächtige Metaphysik des Mentalismus bzw. ein cartesianischer Leib-Seele-Dualismus ist der erklärte Gegner dieser naturalistischen bzw. szientifischen Einheitsidee. Ziel des wissenschaftlichen Naturalismus oder Monismus ist demnach, unsere Reden über Geistiges zu entmythisieren.

Das Projekt der ,Erklärung' der Sprachfähigkeit erhält nun verschiedene Ausprägungen. Quine ,erklärt' die Sprachkompetenz durch Ausbildung gewisser Regularitäten und Dispositionen im sprachlichen und nichtsprachlichen Verhalten im sozialen Kontext. Dieser Regularismus Quines ist am Ende rein deskriptiv. Das heißt, die von Quine präferierte Methode der Einheitswissenschaft ist behavioristisch. Dabei ist der Behaviorismus als diejenige Variante der Denkbewegung des Empirismus zu begreifen, welche in ihrer sinnkritischen Analyse dessen, was es wirklich gibt, nicht, wie der subjektive Idealismus Berkeleys und der methodische Solipsismus Carnaps, bei individuellen Sinneswahrnehmungen oder sogenannten ,Sinnesdaten ' beginnt, sondern bei einem beobachtbaren Verhalten von präsentischen Untersuchungsgegenständen ,at arm's length', wie man sagen könnte.

Chomsky behauptet dagegen, ein behavioristischer Ansatz könne keine ausreichende wissenschaftliche Erklärung dafür liefern, dass nur wir Menschen gewisse rekursive Regeln der Sprachproduktion und des Sprachverstehens und das dafür notwendige syntaktische Formerkennen des ,Parsing ' lernen (können). Vielmehr sei dazu eine besondere Sprach(lern)kompetenz anzunehmen. Diese stellt sich Chomsky nach dem Muster einer automatischen Sprachlernmaschine als eine Art inneres oder angeborenes System parametrisierbarer Regelschemata vor. Durch äußere Einflüsse, genauer: dadurch, dass wir einem gewissen Sprachverhalten anderer Menschen ausgesetzt werden, werden Parameter gesetzt, so dass aus universa- 
len Regelschemata die grammatischen Regeln von Einzelsprachen entstehen.

Chomskys Regulismus und Quines Regularismus stehen sich antagonistisch gegenüber. Dennoch sind beide Ansätze individualistisch in folgendem Sinn: Es wird angenommen, dass der einzelne Mensch Sprache im Grundsatz so erwirbt, wie das auf ganz ähnliche Weise schon Augustinus in seinen Bekenntnissen skizziert und Wittgenstein als Mythos eines angeborenen inneren Denkens kritisiert hatte. Quine nimmt immerhin bloß an, dass sich irgendwie gewisse Regelmäßigkeiten im Sprachverhalten ausbilden. Für ihn wäre es schon zuviel an spekulativer Unterstellung, wenn man interne Regeln annähme. Er betont sogar, dass alle derartigen Regeln durch das reale Verhalten der Individuen wesentlich unterbestimmt bleiben. Mit anderen Worten, es gibt keine grammatischen Regeln, es sei denn in den viel später von uns zu bestimmten Zwecken verfassten Sprachbüchern und Übersetzungsmanualen. Diese aber sind als solche keineswegs schon durch den faktischen Sprachgebrauch determiniert, sondern gehen als Konstruktionen über diesen hinaus. Diese Indeterminiertheit der in Sprachlehrbüchern ,explizierten' syntaktischen und semantischen Regeln bzw. der durch diese (re)konstruierten grammatischen Formen und Bedeutungen zeigt sich schon daran, dass es viele wesentlich verschiedene derartige Manuale geben kann, die zum (je bisherigen faktischen) Sprachverhalten gleich gut passen. Formal ist dieses Argument Quines (sozial)behavioristisch im Sinne des Empirismus Humes. Zugleich ähnelt es, ohne dass das je klar gemacht wäre, Poppers Einsicht in die Unlösbarkeit des Induktionsproblems: Die von uns gebastelten Theorien zur Darstellung der Phänomene, auch der Phänomene des Sprachverhaltens, sind durch diese Phänomene nie eindeutig bestimmt.

Chomsky dagegen meint, dass die Tatsache der überwältigenden Übereinstimmungen in unseren Urteilen der Wohlgeformtheit sprachlicher Ausdrücke nur erklärbar sei, wenn wir annehmen, dass im Spracherwerb Regeln der Sprachproduktion und der Sprachformerkennung (des Parsing) gelernt werden, welche für unendlich viele Fälle die (syntaktische) Wohlgeformtheit bestimmen und die es daher in einer kognitionstheoretischen Theorie darzustellen und in ihrer Genese zu erklären gilt.

Davidson, obwohl Schüler Quines, stellt sich im Grunde auf die Seite Chomskys. In seinem Projekt geht es nämlich gerade darum, theoretisch zu erklären, wie aufgrund endlich vieler Beispiele unendlich viele Ausdrücke und Sprechhandlungsformen gelernt werden können, und was es heißt, dass diese am Ende ,korrekt` oder ,richtig' verstanden werden. Dazu be- 
nutzt Davidson, ohne dass das wirklich völlig zureichend explizit gemacht wäre, die Idee der logischen Form als eines (tiefen)syntaktischen Aufbaus der Ausdrücke. Diese Idee geht auf Russell und Wittgenstein zurück. Bei Davidson hat man sie sich von der Art vorzustellen, wie sie von Chomsky mit starker Betonung der Eigenständigkeit des Syntaktischen, von Montague aber in enger Anlehnung an Logiksprachen entworfen wurden. An einem solchen Aufbau (einer ggf. die Oberflächenausdrücke disambiguierenden ,logischen Form') orientiert sich Davidsons Verwendung von Freges semantischem Kompositionalitätsprinzip für die Rekonstruktion des Aufbaus von Wahrheitsbedingungen der zu analysierenden Sätze und Aussagen.

Davidson Idee ist nun, grob skizziert, die, dass sich der Hörer in der Rolle eines sogenannten radikalen Interpreten befindet. Das ist im Wesentlichen gerade die Situation, die Augustinus in seinen Bekenntnissen für den Spracherwerb des Kindes schildert. Der radikale Interpret muss dazu, erstens, die gehörten Sätzen S (der Oberflächensprache) a priori als richtig und wahr annehmen. Er muss als Hörer davon ausgehen, dass die Sprecher etwas Richtiges tun bzw. etwas Wahres sagen. Denn sonst kommt das Verfahren der Deutung des Gesagten durch eine Rekonstruktion seiner Richtigkeits- oder Wahrheitsbedingungen in einer radikalen Interpretation gar nicht in Gang. Es ist daher durchaus irreführend, diesen Beginn der radikalen Interpretation mit dem Titel „Prinzip der Charity“ zu überschreiben. Denn ein derartiges Nachsichtigkeitsprinzip kann als solches erst auf der höheren Ebene der bewussten Interpretation, Deutung oder Übersetzung einer Sprache in eine andere gebraucht werden. Auf der basaleren Ebene, auf der wir Sprache zu verstehen und zu gebrauchen lernen, geschieht Anderes. Eben das hat Wittgenstein gesehen, als er Augustinus kritisierte, der den Spracherwerbsprozess wie Davidson als den Erwerb einer Interpretationsmethode, also einer Methode der Übersetzung in eine schon vorhandene Sprache des Denkens schildert.

Bei Davidson wird dabei dem gehörten Satz S eine Beschreibung einer logischen Tiefenstruktur $S^{*}$ zugeordnet, und zwar so, dass nach den logischen Kompositionsregeln diese Satzform $\mathrm{S}^{*}$ den Wert das Wahre zugeordnet erhält genau dann, wenn man zusätzlich gewisse ,kausale ' Bezüge auf die Welt etwa für (implizit oder explizit) deiktische Wörter und Anaphora unterstellt (modulo notwendiger Disambiguierungen). Dies alles erhält bei Davidson die Form einer Tarski nachempfundenen formalen Wahrheitsbedingungensemantik. Tarski hatte nämlich (übrigens auf strukturell ähnliche Weise wie zuvor schon Gödel) die rekursiven Regeln der semanti- 
schen Deutung parallel zur tiefensyntaktischen Analyse in gewissem Sinn zu kalkülisieren versucht. (Es sollte, genauer, eine Erfüllungsrelation zwischen einem Modell und den Sätzen, also den geschlossenen Formeln, einer ,Objektsprache" in einer axiomatisch-deduktiven Rahmentheorie vollformalisiert werden.) Tarskis berühmt-berüchtigtes T-Prinzip oder Wahr-

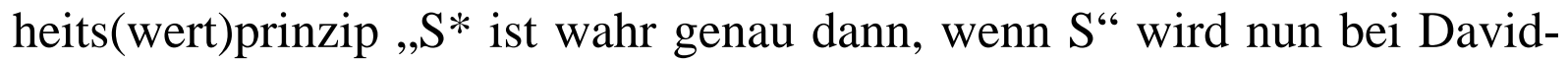
son als eine Art Kriterium dafür benutzt, dass $\mathrm{S}^{*}$ eine dem Satz S (bzw. der zugehörigen Äußerung) angemessene Tiefenstrukturbeschreibung ist (wobei wir wieder beachten müssen, dass die Oberflächensprache im allgemeinen quantorenlogisch ambig ist).

Mir geht es hier nicht um eine detaillierte Darstellung der Semantiktheorie Davidsons. Mir geht es darum zu zeigen, dass gerade auch sie dem Augustinischen Bild des individualistischen Spracherwerbs durch interpretative Rekonstruktion einer ,Bedeutung' verhaftet bleibt, auch wenn die Rede von der Bedeutung aufgelöst wird in einen deduktionslogischen oder formalinferentiellen Umgang mit Sätzen S und (Beschreibungen von) logischen Satzformen $S^{*}$. Dabei wird einfach unterstellt, dass die Bedeutung bzw. formale Gebrauchsweise der logischen Formworte „und“, „nicht“ „N=M" und „für alle $\mathrm{x}$, so dass $\mathrm{A}(\mathrm{x})$ “" schon gelernt bzw. bekannt ist. Denn es bilden bei Davidson die deduktiven Schlussregeln der Prädikatenlogik der ersten Stufe (mit Gleichheit) ein nicht weiter analysiertes, sondern stillschweigend und a priori vorausgesetztes, Rückgrat der formalen Semantiktheorie.

\subsection{Normen des Richtigen in der einspruchsfreien Anerkennung}

Robert Brandoms sprachphilosophischer Ansatz versucht nun in gewissem Sinn, die Einsichten der Ansätze Quines und Davidsons mit der Empirismuskritik von Sellars und der Kritik Dummetts an Davidson abzugleichen. Dummett zufolge ist Davidsons Semantiktheorie deswegen , allzu bescheiden', weil sie nur zeigt, wie man zu einer gegebenen Sprache einen formalen Wahrheitsbegriff konstruieren kann, wenn man den Umgang mit den Regeln der Tiefenstruktursprache (also mit den Strukturbeschreibungen $\mathrm{S}^{*}$ ) in Bezugnahme auf die Oberflächensprache (die gehörten Sätze S) schon beherrscht. Wie man das aber lernen können soll, wird von Davidson überhaupt nicht erläutert. Das aber müsste eine ,full blooded theory ' der radikalen Interpretation bzw. eine entsprechende ,erklärende' Semantiktheorie zeigen, wenn sie denn das Ziel erfüllen sollte, das ihr von Davidson (und anderen Autoren) selbst gesetzt ist. 
Bei Brandom geht es nun darum, sowohl das Unbefriedigende eines sozialbehavioristischen Regularismus, als auch das eines spekulativen Regulismus angeborener Sprach(erwerbs)regeln zu überwinden. Dabei verfolgt Brandom zugleich ein Programm der Logikbegründung. Eine solche wird in der Tat nötig, wenn man das System der deduktiven Inferenzregeln etwa der Prädikatenlogik nicht, wie im Grunde bei Davidson, einfach als quasi angeborene logische Maschine annehmen möchte. In Brandoms Programm des Making It Explicit ergeben sich vielmehr Regeln erst aus einem Prozess der analytisch-rekonstruktiven Explikation eines impliziten (oder nach Karl Bühler besser: empraktischen) Know-how ${ }^{2}$. Regeln sind dabei, ähnlich wie bei Wittgenstein, a fortiori explizit. Es gibt sie nur zusammen mit einem Regelausdruck. Das, was das richtige Tun in Ausführungen eines Know-how bestimmt, wird unter dem Titelwort „Norm“ artikuliert. Gerade auch für die Anwendung einer Regel, verstanden als Regelausdruck, bedarf es der Norm bzw. des Know-how ihres rechten Gebrauches.

Inferentielle Regeln explizieren demgemäß praktisch schon beherrschte Normen des richtigen Umgangs mit Sprache. In gewissem Sinn haben das Wittgenstein und Quine schon so gesehen. Diese Regeln werden daher nicht, wie im Regulismus Chomskys und bei Davidson (jedenfalls dann, wenn man deren Ausführungen radikal genug liest), nach Art eines deus ex machina im Gehirn der einzelnen Menschen unterstellt.

Brandom erkennt nun insbesondere, dass der basale Begriff, den es zu erläutern gibt, der soziale Begriff des Richtigen und damit der Begriff der empraktischen Normativität ist. Im Fall der Syntax ist es der Begriff der Richtigkeit des Ausdrucks, im Fall der Semantik besonders der des richtigen Schlusses, der korrekten Inferenz. Das ist der Grund, warum Brandoms Semantik zu Recht den Titel „Inferentialismus“ trägt. Dieser Inferentialismus ist ein Normativismus aus folgendem Grund: Brandom erklärt den Begriff des Richtigen weder, wie der Regulismus, durch hypostasierte Regeln, noch, wie der Regularismus, durch bloß kontingente Verhaltenskoordinationen, sondern eben durch empraktische Normen, die man zunächst praktisch beherrscht und dann erst, partiell, in der Form von Regeln oder

\footnotetext{
${ }^{2}$ Dabei spreche ich von einem empraktischen Können später auch von einer empraktischen Anerkennung oder einer empraktischen Norm, wenn auf ein Verhalten oder ein Knowing-how gerade auch im intentionalen Handeln Bezug genommen wird, das durch eine gemeinsame Praxis geformt ist. Manches daran kann über eine Formanalyse in ein explizites und reflektiertes Wissen über die Praxis, über ihre Formen und Normen, und damit in eine explizite Anerkennung dieser Formen und Normen überführt werden.
} 
Sätzen explizit machen kann. Dabei haben diese Normen selbstverständlich einen sozialen Status. Sie existieren im sozialen Verhalten der Teilnehmer. Sie gehören zur Form eines Könnens. Dieses zeigt sich in der empraktischen Teilnahme an der entsprechenden gemeinschaftlichen Praxis. Diese kann sich als solche nicht einfach aus konventionellen Koordinationen ichintentionaler Akte ergeben, wie das David Lewis in seinem wichtigen Buch „Conventions" meint. Denn man kann gar nicht voraussetzen, dass schon alle Teilnehmer der Praxis die Handlungsformen des Beabsichtigens, der rechten Verfolgung von Absichten und damit des ich-intentionalen Handelns beherrschen.

Was Verstehen ist, das muss also zunächst erläutert werden über die kompetente Teilnahme an einer entsprechend gestuften normativen Praxis. In gewissem Sinn geht es dabei darum, Wittgensteins spätere Einsicht, dass Verstehen kein Interpretieren ist, sondern in der Fähigkeit der rechten Teilnahme an einer menschlichen Lebensform besteht, explizit zu machen. ${ }^{3}$ Dabei erlaubt eine zunächst noch eher diffuse Praxis des richtigen gemeinsamen Verhaltens und empraktischen (,impliziten") Könnens gewissermaßen erst sekundär eine explizite Zuschreibung von inferentiellen Verpflichtungen und Erlaubnissen.

Robert Brandom verwandelt in seinen Überlegungen Davidsons Einsicht in die besondere Rolle des Wahren gewissermaßen in die folgende ,hegelianische' Einsicht: Das Richtige ist immer nur als ein Ausbleiben von Unrichtigem bestimmt, also immer nur soweit, als kein Widerspruch auftritt. Ein solcher Widerspruch zeigt sich etwa in einem Sanktionsbenehmen, später dann auch in einem bewussten und seinerseits auf Richtigkeit kontrollierten Sanktionshandeln. Der Widerspruch wird also manifest in einem Tadel oder Einwand. Er wird als solcher zunächst unmittelbar verstanden, nämlich als Zeichen dafür, dass etwas an der Gemeinsamkeit im Tun defizient ist. Damit wird, so würde ich den Gang der Überlegung grob skizzieren, wie bei Hegel die Anerkennung zur Grundlage jeder Bewertung des Richtigen und damit zum Grund der Existenz von Normen. Anerkennung selbst besteht dabei darin, dass die Gemeinsamkeit im Tun nicht infrage gestellt wird, etwa aktiv durch einen Widerspruch oder durch eine bloße Abkehr vom ,gemeinsamen Spiel'. Ganz grob könnte man daher, in gewissem Sinn mit Brandom, sagen, dass Anerkennung im Aus-

\footnotetext{
${ }^{3}$ Dabei zeigen frühere Texte, in denen das Verstehen noch als Angabe verbaler Erklärungen und damit als ein Übersetzen gedeutet wird, wie schwer gerade auch Wittgenstein selbst diese Einsicht gefallen ist.
} 
bleiben von einem möglichen negativen Sanktionsverhalten oder Sanktionshandeln besteht. ${ }^{4}$

Bewusste Anerkennungen, wie sie bei Axel Honneth diskutiert werden, sind längst schon als intentionale Handlungen bzw. als hochstufige Haltungen zu Personen aufzufassen. Hegels Einsicht in die Rolle empraktischer Anerkennungen von Normen und Formen des Richtigen für die Bildung intentionaler Autonomie liegt auf einer anderen, tieferen, Ebene: Es geht um die implizite Anerkennung von Richtigem, um die empraktische Kompetenz des Verstandes, das Richtige zu tun oder zu sagen. Dieses Können ist jeder Kompetenz der Vernunft vorgelagert, autonom darüber zu befinden, was richtig ist oder als richtig gelten soll. Im sogenannten Kampf um Anerkennung geht es bei Hegel, anders als bei Honneth, nicht um einen Kampf zwischen Personen. Es geht nicht um eine explizite, moralische, Anerkennung von Personen. Es geht um eine praktische Anerkennung impliziter Formen und Normen des Richtigen. Im Einzelfall geht es insbesondere um eine intrapersonale Auseinandersetzung zwischen unmittelbaren, rein subjektiven, Begierden auf der einen Seite, der Bestimmung des richtigen Tuns durch gemeinsame Normen auf der anderen, also um den Kampf zwischen dem ,Leib“ als dem Knecht und dem ,Geist", der nur Herr bleibt, wenn die Person als ganze das Richtige tut.

In gewissem Sinn lässt sich im Anschluss daran auch die Idee von Wilfrid Sellars, dass sich im Geben von und Fragen nach Gründen Inhalte zeigen, auf eine interessante Weise weiter entwickeln. Denn das Fragen nach Gründen für ein Urteil oder eine Handlung, eine Aussage oder für sonst einen Sprechakt, ist immer ein Fragen nach normativen Berechtigungen. Damit wird eine Frage, jedenfalls in einem gewissen Sinn, als ein potentieller Einwand begriffen, den es in der Antwort, im Geben von Gründen, auszuräumen gilt.

Die zugrundeliegende Strukturidee des normativen Inferentialismus Brandoms ist also, grob skizziert, diese: Das semantisch Richtige manifestiert sich in der dialogischen Situation des Sprechens und Hörens, Handelns und Anerkennens empraktisch immer nur insoweit, als sich Sprecher und Hörer mit dem inferentiellen Benehmen des jeweils anderen zufrieden geben, dieses also faktisch nicht sanktionieren. Ich spreche hier, in meinem

\footnotetext{
${ }^{4}$ Die entsprechende Grundeinsicht Hegels, dass die Anerkennung von Richtigem im Ausbleiben von Einsprüchen besteht, und wie sie zur Konstitution von Begriffen und Inhalten über differentielle und inferentielle Richtigkeiten beiträgt, hat Robert B. Brandom in seinem wichtigen Buch Making It Explicit. Reasoning, Representing and Discursive Commitment (hier: Brandom 1994) weiter entwickelt.
} 
etwas idiosynkratischen Differenzierungsversuch, von einem inferentiellen Benehmen, weil es noch nicht unbedingt ein bewusst kontrolliertes intentionales Handeln sein muss, dessen Form schon vom Handelnden explizit artikuliert werden könnte, aber auch nicht bloß ein dispositionelles Verhalten ist. Auch die Anerkennung, die sich implizit oder empraktisch darin zeigt, dass sich die Teilnehmer der teils sprachlichen, teils nichtsprachlichen Interaktion mit dem, was die anderen tun, zufrieden geben, ist im allgemeinen Fall ein Benehmen, also nicht immer schon ein bewusstes und intentionales Handeln.

Dass gerade im Sozialen das Richtige zunächst rein negativ zu charakterisieren ist, unabhängig von der Begleitung durch subjektive Intentionen und Meinungen, das ist eine tiefe Einsicht. ${ }^{5}$ Dabei ist weder ein zufälliges Sanktionsverhalten, noch ein schon intentionales Sanktionshandeln, sondern gerade die empraktische Fortsetzung ,gemeinsamer Spiele' bzw. das Ausbleiben eines Sanktionsbenehmens entscheidend für den Realbegriff des Richtigen. Das ist gerade auch deswegen von solcher Bedeutung, weil nur damit der Freiheitsspielraum des Richtigen angemessen begreifbar wird.

Das Ausbleiben von Einsprüchen bestimmt also, so lese ich Brandom, was faktisch und praktisch im und durch das Sozialverhalten als richtig oder erlaubt bewertet oder eben anerkannt wird. Die Frage, was anerkennungswürdig und damit in einem entwickelteren Sinn richtig ist, das ist unbedingt als höherstufig und in der methodischen Ordnung ,später ${ }^{6} \mathrm{zu}$ begreifen. Das kann man relativ schnell einsehen, wenn man bedenkt, dass auch diese Urteile über Anerkennungswürdigkeiten faktisch gefällt und faktisch anerkannt werden müssen.

\subsection{Von Konventionen zu Intentionen}

Selbstverständlich ließe sich zu diesem Ansatz noch viel mehr im Detail sagen. Im Folgenden interessiert mich das Problem, dass sich bei Brandom das Reich des Normativen, der Anerkennungen und Nichtanerkennungen, aus dem faktischen Sozialverhalten der einzelnen Individuen ergeben soll. Zentral ist dabei das Sanktionsverhalten gegenüber Personen, deren Tun

\footnotetext{
${ }^{5}$ Schon der große Ethnologe Frazer, dessen Golden Bough Wittgenstein bekanntlich zum Ausgangspunkt seiner Reflexionen auf die Rolle von Mythen nimmt, schreibt: „Für die Gesellschaft ist das Verhalten wichtig, nicht die Meinung: Wenn nur unser Handeln gerecht und gut ist, so kümmert es die anderen keinen Deut, ob wir mit unseren Ansichten im Irrtum sind“. Frazer, 82, zitiert nach Hayek 1996, 167.
} 
uns jeweils einzeln ,irgendwie nicht gefällt" - bzw. das Ausbleiben eines derartigen Sanktionsverhaltens.

Dabei scheint der methodische Individualismus und die evolutionistische Erklärungsstrategie, die Brandom verfolgt, zunächst ganz plausibel zu sein. Denn es muss sich doch das Phänomen der besonderen geistigen oder kognitiven Vermögen der Menschen, insbesondere aber des Sprachvermögens, damit auch das (laute oder leise) planende Denken, vor dem Hintergrund einer evolutionsbiologisch erklärbaren Entwicklung des Verhaltens der einzelnen Individuen ergeben. In der realen Wirklichkeit gibt es, so scheint es also, nur die Individuen und ihr Verhalten. Unsere Reden über den Geist, die Seele oder gar den freien Willen, zum Beispiel, sind doch bestenfalls als figurative façon de parler zu begreifen, die, wenn sie überhaupt sinnvoll sein sollen, , grundsätzlich“ immer auf die eine oder andere Weise zurückführbar sein müssen auf das Verhalten der Individuen. Analoges gilt für Wittgensteins vage Rede über eine menschliche Lebensform, oder für unsere Reden über Institutionen, Praxisformen oder sonstigen Formen gemeinsamen oder dann auch individuellen Handelns. Es gilt schließlich auch für unsere Reden über Rollen und am Ende auch über personale Kompetenzen. Demnach sollten auch die Gegenstände reflektierender Rede, wie etwa der Verstand, die Vernunft oder der menschliche Geist nicht hypostasiert werden. Sie verweisen auf keinen Fall auf eigenständige Akteure hinter den Personen. Das wäre Animismus oder Mentalismus. Sie sind vielmehr als Aspekte personaler Kompetenz zu verstehen.

Aber auch unsere Reden über Kollektivsubjekte wie den typischen Deutschen, den deutschen Soldaten usw. sind generisch zu verstehen. Das gilt etwa auch für jede Rede über den Status bzw. die Rolle eines Präsidenten in einem Land oder einem Verein. Das Beispiel zeigt, wie wir hier auf (Teil-)Formen gemeinsamer Praxis verweisen und von den individuellen Besonderheiten der Einzelpersonen absehen. Derartige Reden über Rollen, Teilformen und Normen einer Praxis betreffen also nicht einfach das rein individuelle Tun. Würden wir bloß auf das Einzelne fokussieren, so würde dessen Abhängigkeit von einem allgemeinen Rahmen gerade nicht angemessen begreifbar werden. Dem korrespondiert Hegels etwas kryptische Aussage, dass der Geist nicht bloß Objekt unserer Reflexion ist, sondern auch als Subjekt zu begreifen ist. Dabei ist die Gefahr der Hypostasierung derartiger ,Großsubjekte' ebenso zu vermeiden wie eine allzu große Skepsis gegen entsprechende Redeformen, da sonst auch sinnvolle Rede über 
die Gesellschaft, über Institutionen und Praxisformen infrage gestellt würden. ${ }^{6}$

Es geht dann aber auch um Anderes als bloß um das rechte Verständnis von sprachlichen Reflexions- und Ausdrucksformen. Es geht unter anderem um die folgende Frage: Wie lässt sich begreifen, dass gerade die Gemeinschaft und eine durch sie erzeugte Art eines ,unbewusst-gemeinschaftlichen" Verhaltens es uns allererst ermöglicht, als individuelle Person irgendwie bewusst intentional zu handeln? Wie werden also gemeinschaftliche Normen des Richtigen in Konventionen inferentiellen Benehmens zur Bedingung der Möglichkeit ,autonomen' Urteilens und Tuns? Und wie verhält sich diese Tatsache zur Möglichkeit des bewussten kooperativen Handelns? Die Fragen unterstellen, dass das, was es zu begreifen gilt, real ist, also dass die folgenden Thesen richtig sind:

a) Gemeinschaftliches Verhalten ist die Grundlage für individuelles Bewusstsein, damit auch für die Möglichkeit intentionalen und zweckgerichteten Handelns, also für jede Form planender und handelnder Selbstbestimmung und Autonomie.

b) Wir müssen dazu zwischen einem schon subjektiv bewussten Anerkennen bzw. einem (ich-)intentionalen kooperativen Handeln und einer vorbewussten gemeinschaftlichen Anerkennen und Tun unterscheiden.

c) Das vorbewusste Anerkennen ist ein gemeinschaftliches Verhalten.

Ein zentraler Punkt in diesem Überlegungsrahmen betrifft die Rolle der Sprache als Möglichkeitsbedingung für das freie Handeln einerseits, für personale Autonomie andererseits. Es geht, genauer, darum, wie symbolische Repräsentationen von Handlungen und Handlungsfolgen möglich werden und in welchem Sinn sie Bedingungen für bewusste Absichten sind. ${ }^{7}$

Jedes Sinnverstehen scheint am Ende gerade gemäß einer Gebrauchstheorie der Bedeutung bzw. des Sinnes von Wörtern, Sätzen und

\footnotetext{
${ }^{6}$ Im Grunde sieht das, nur um ein Beispiel zu nennen, auch Niklas Luhmann: Der methodische Individualismus würde jede Reflexion auf Gemeinschaft und Gesellschaft unmöglich, weil sprachlos machen, jedenfalls wenn man ihn allzu ernst nähme. Eine Gesellschaftstheorie würde dann erst recht unmöglich.

${ }^{7}$ Das eben besagt das unserer Überlegung vorangestellte Motto. Es lautet in der ebenfalls bei von Hayek zu findenden Langform des Konfuzius so: "Wenn die Bezeichnungen unrichtig sind, stimmt die Sprache nicht mit der Wahrheit der Dinge überein. ... Die Menschen werden keinen Halt für Hand und Fuß haben“7 . Es sagt in dieser Form mehr, als dass Sprachmissbrauch zu Orientierungsproblemen führt. Es sagt, positiv, dass Freiheit und Autonomie von einem richtigen Sinnverstehen abhängen.
} 
Sprechhandlungen in nichts anderem zu bestehen als in einer Art des ,normativ richtigen' Benehmens, also der Einhaltung von praktischen Inferenznormen. Deren Existenzweise scheinen wiederum am Ende die komplexer ,Konventionen“ eines Sprach- und Sozialverhaltens zu sein. Dabei sind diese Konventionen allerdings noch nicht, wie bei David Lewis, als ichintentionale, also jeweils individuell und zweckrational durchdachte, Lösungen von Koordinationsproblemen zu verstehen, sondern existieren bloß in einer normativen Praxis des sozialen bzw. gemeinschaftlichen Verhaltens - inklusive der zugehörigen (Benehmens-)Praxis der Anerkennung von (inferentiellen) Richtigkeiten und der (empraktischen) Nichtanerkennung von Unrichtigkeiten im Einspruch gegen (empraktische) Normverletzungen.

Wie soll sich dann aber aus der bloßen Beherrschung des konventionell Richtigen personale Autonomie ergeben können? Die Frage steht in enger Analogie zur Frage, wie sich durch disziplinierende Abrichtung die Möglichkeit des freien Handelns und der Selbstbestimmung ergeben soll. Eben das hat Kant behauptet und auch Wittgenstein scheint dies sagen zu wollen. Woher aber wissen wir dann, dass die von Kant „Autonomie“ genannte Selbstunterwerfung nicht in Wirklichkeit eine Unterwerfung unter vorgegebene Schemata des Verhaltens ist, wie Nietzsche keineswegs zu Unrecht erwägt? Eine völlig analoge Frage richtet sich an Wittgenstein, dem zufolge wir Sprache und andere Praxisformen ja ebenfalls durch Abrichtung lernen. Ist dieses Lernen wirklich analog zur Abrichtung eines Hundes? Wenn das nicht der Fall sein sollte, worin bestehen die wesentlichen strukturellen Unterschiede?

Wie also sind individuelle Intentionen möglich, wenn sie auf allgemeine Normen des Richtigen im inferentiellen Verhalten aufruhen sollen? Wie funktioniert das ,boostrapping', der Münchhausentrick, sich am eigenen Schopf aus dem Morast der bloßen Gewohnheit zu befreien? Wie werden wir aus konventionellen Kollektiv- und Gemeinschaftswesen zu selbständigen Individuen? Wie verwandelt sich die Anpassung im Verhalten an Normen in ein freies Handeln? ${ }^{8}$ Wie ist freies Urteilen und autonomes Handeln überhaupt möglich? Wir brauchen offenbar eine angemessene, überzeugende, Geschichte, die zeigt, dass und wie eine Entwicklung zum freien Urteilen und autonomen Handeln im Ausgang von der Befolgung von inferentiellen Normen möglich wird. Wir brauchen ein Verständnis des Münchhausentricks oder des boostrappings, das zeigt, wie individuelle In-

\footnotetext{
${ }^{8}$ Die Normen gibt es, man erinnere sich, ihrerseits nur in der Form eines entsprechenden Sanktionsverhaltens der anderen bei Normabweichungen.
} 
tentionalität möglich wird. Die Frage ist, ob Brandom uns die Umrisse einer solchen Analyse skizziert oder ob seine Skizze in wesentlichen Punkten mangelhaft ist. Die Frage ist nicht, ob das theoretische Bild vollständig ausgemalt ist, ob man also der Skizze noch viele nette Details hinzufügen kann. Es geht um die Gesamtform der Skizze, und um die Gesamtorientierung, die sie gibt. Ist diese im Wesentlichen anerkennenswert, also in relevanten Bereichen richtig? Hier kann ich dazu nur eine möglichst charitable Lesart entwickeln, die durch eigene Interpolationen und $\mathrm{Zu}$ rechtstellungen zeigt, warum an dem Ansatz vieles nachhaltig interessant bleiben wird.

\section{Lerntheoretische Voraussetzungen personaler Kompetenzen}

\subsection{Gemeinsame Weltbezüge}

Zunächst ist dazu die Form eines Anerkennungsbenehmens in der Form eines noch nicht von allen Beteiligten bewusst bzw. explizit kontrollierten gemeinsamen Tuns zu skizzieren. Wenn etwa eine Mutter einem Kind oder dieses der Mutter etwas zeigt, so koordinieren beide, wenn das Zeigen glücken soll, ihr Verhalten auf gewisse Weise. Falls etwa ein Beteiligter unaufmerksam ist, protestiert der andere. Wenn es keinen derartigen Widerspruch gibt, wird das Tun aller Beteiligten faktisch als richtig anerkannt. Falls das kooperative Benehmen überraschend gut klappt, kann es zu einem besonderen Lob kommen.

Die Idee ist nun die, dass schon die Deixis eine gemeinsame Verhaltensform oder Wir-Handlungsform $\mathrm{H}$ ist, die über gewisse Rollenverteilungen $\mathrm{H} 1, \mathrm{H} 2, \mathrm{H} 3$ etc. ... definiert und als solche kontrolliert wird. Sie und nicht etwa der räumliche Ort der Person allein, definiert das, was wir die Perspektiven der Akteure nennen könnten. Mit anderen Worten, in einem sogenannten Perspektivenwechsel von mir zu dir fühle ich mich nicht etwa hinein, wie es wäre, du zu sein, oder die Welt von dir aus wahrzunehmen. Wir reden zwar so. Aber wir meinen damit, dass wir im gemeinsamen Leben verschiedene Rollenspiele zu beherschen gelernt haben. Das Zeigen selbst ist ein solches Rollensspiel. Ohne die Ergänzung durch die Reaktionen dessen, dem etwas gezeigt wird, bliebe es ganz unverstanden. Hinzu kommt die Kontrolle, ob dieser grundsätzlich angemessen, wenn vielleicht auch noch irgendwie falsch bzw. unrichtig, weil irgendwie noch auf zu korrigierende Weise, oder schon ganz und gar richtig und damit zur vollen Zufriedenheit des Zeigenden reagiert, Mit anderen Worten ein Zeigen H1 
ist immer nur als Teilmoment einer gemeinsamen Handlung $\mathrm{H}$ mit $\mathrm{H}=(\mathrm{H} 1, \mathrm{H} 2, \mathrm{H} 3 \ldots)$ zu verstehen. $\mathrm{Zu} \mathrm{H}$ gehört also wesentlich auch die handelnde Reaktion $\mathrm{H} 2$ der zweiten Person und das Anerkennungshandeln bzw. Anerkennungsbenehmen H3 der ersten Person. Dabei kann die Reaktion $\mathrm{H} 2$ in einer verbalen oder noch nicht verbalen Handlung oder einem entsprechenden Benehmen bestehen, aber auch in einer Art Nachfrage. Die Kontrollhandlung H3 kann im Fall, wenn das Zeigehandeln offenbar gescheitert ist, seinerseits weitere Korrekturhandlungen auf Seiten der ersten Person und damit weitere Reaktionen auf Seiten der zweiten nötig machen.

Jeder von uns kennt diese Rollenspiele sowohl aus der Perspektive der ersten als auch der zweiten Person, so wie wir wissen, was es heißt, Sprecher oder Hörer zu sein. Das heißt wir können die Rollen abwechslungsweise so spielen, wie ich zum Beispiel Verkäufer oder Käufer sein kann. Empraktisch, also im Modus des know-how, wissen wir, was das heißt. In eben diesem Sinn weiß ich, was es heißt, dass mir jemand etwas von vorne zeigt, was er, aber nicht ich, von hinten sieht - und umgekehrt. Das heißt, diesen nichttrivialen Perspektivenwechsel beherrschen wir Menschen, wenn oder weil wir die entsprechenden gemeinsamen Zeigespiele beherrschen. Und wir beherrschen sie nicht etwa erst dann, wenn wir uns ,mental vorstellen' (können), wie es wäre, die anderen Rollen zu spielen, wenn wir also sprachliche oder symbolische Repräsentationen für die Personen und Rollen etwa in stillen Selbstgesprächen zur Verfügung haben, sondern schon dann, wenn wir die Rollen einfach wirklich spielen können.

Um zu sehen, dass dem so ist, könnte folgende Erinnerung weiterhelfen. Wir bemerken schon als sehr kleine Kinder, wenn jemand zunächst an einem gemeinsamen Spiel, gerade auch einem Zeigespiel, hinreichend mitspielt und dann vielleicht mehr und mehr solitär wird, also nur noch für sich spielt. Man könnte, wenn man wollte, im Detail testen, woran sich zeigt, dass Kinder das bemerken, und wie sie dabei reagieren. ${ }^{9}$

Die in den Beispielen angezeigte Unterscheidung zwischen gemeinsamen Spielen und Solitärspielen hängt eng zusammen mit Raimo Tuomelas Unterscheidung zwischen handlungstheoretischen Individualbegriffen oder i-Begriffen und Gemeinbegriffen oder g-Begriffen, bzw. zwischen einem Ich-Modus und einem Wir-Modus eines Tuns sowohl qua Verhalten als

\footnotetext{
${ }^{9}$ In Wittgensteins Liste für Spiele treten entsprechende Beispiele für gemeinsame Spiele oder Nicht-Solitärspielen und Solitärspiele auf: Wir spielen zum Beispiel zunächst zusammen mit zwei Bällen und einer zieht sich mehr und mehr zurück, spielt nur noch mit seinem Ball, nicht mehr mit der 2. Person. Oder wir tanzen zunächst zusammen und am Ende tanzt jeder für sich.
} 
auch qua Handeln. Der Unterschied liegt darin, dass sich die ersteren auf ein individuelles Tun und Handeln, die letzteren auf ein gemeinsames Handeln beziehen. Spreche ich etwa von meinem Wunsch und meiner Maxime, so sind dies i-Begriffe, spreche ich von unserer gemeinsamen Absicht und unserer Zusammenarbeit, so sind dies g-Begriffe.

Der Zusammenhang von gemeinsamen Spielen H und der Möglichkeit zum Perspektivenwechsel und damit der Möglichkeit zu einem intentionalen und objektiven räumlichen Bezug auf Zuhandenes und dann auch Vorhandenes im präsentischen Anschauungsraum ist - trotz der bekannten allgemeinen Hinweise Heideggers auf das Existential des ,Mitseins' ${ }^{6}$ - noch kaum zureichend begriffen. Meine zugehörige These ist, dass erst im Perspektivenwechsel, also im Vergleich zwischen komplexen Teilhandlungen H1 und H2, und keineswegs ,rein optisch", oder gar schon über irreale Konditionalaussagen oder mystischen Einfühlungen bemerkt werden kann, dass ich hier und nicht dort, ich und nicht du bin - wie man das wohl sagen muss, da ,ich“ und „du“ Differenzierungen eines diffusen Wir artikulieren. Diese Differenzierungen geschehen empraktisch viel früher als die Verwendungen der Wörter ,ich“ und ,du“.

Das diffuse Ich/Wir - als eine Art Teilmoment des Daseins Heideggers - ist allerdings ein tiefes Problem. Gemeint ist eine Haltung in einer präsentischen Interaktion, in der, wie z.B. bei Kindern, in gewissem Sinn noch keine bewusste und explizite Ich-Du oder gar Ich-Du-Er-Es-WirDifferenzierung vorgenommen (oder gar beherrscht) ist, so dass also in gewissem Sinn das reflektierte Meta-Wissen um die Perspektivität noch fehlt oder noch partiell defizitär ist. Das kann sich etwa darin zeigen, dass noch partiell unklar bleibt, warum Andere gerade wegen ihrer differenten Perspektive etwas gar nicht tun können. ${ }^{10}$

Im Detail gibt es hier noch vieles genauer zu artikulieren, um am Ende eine gewisse Übersicht zu erhalten darüber, was einer so alles verstehen muss, um im vollen Sinn ,ich“ und „du“ und „,wir“ sagen zu können, also nicht bloß die Wörter sagen kann, sondern deren ,Grammatik' beherrscht. Es geht dabei keineswegs nur um das implizite Wissen um seine eigenen leiblichen Grenzen, sondern gerade um den Perspektivenwechsel als Rollenwechsel. Es geht dann, genauer, auch um die Entwicklung eines empraktisch manifesten und dann auch expliziten (verbalen) Wissens um die

${ }^{10}$ Man denke zum Beispiel daran, dass nicht alle rein logisch möglichen Wir-Spiele wirklich funktionieren, weil die anderen das einfach nicht tun können, was ich gern möchte, was also meine Spielidee verlangen würde. Kinder verlangen entsprechend von Eltern oft Unmögliches. 
Probleme dieses ,Wechselns ', dass nämlich der andere anders ist, etwa seine eigenen Grenzen hat.

Sprechen und Hören, etwas sagend Meinen und etwas vom Gesagten Verstehen sind also jeweils Teilaspekte eines kooperativen Spiels. Das Erfragen und die Angabe von Gründen kommt da erst sehr spät hinzu. Daher ist Bedeutung und Verstehen nicht auf das Geben und Nehmen von expliziten Gründen oder das Zuschreiben von Inhalten reduzierbar. Hier scheint zumindest Sellars, vielleicht auch noch Brandom, schon viel zu viel an Verständnis vorauszusetzen. Wenn wir die zentralen logisch-methodischen Ordnungen in der Sprachkompetenz genauer als bisher begreifen wollen, ist vielmehr das Folgende zu tun: Es ist das Koordinations- und Kooperationsspiel des Sagens und Tuns, des Protestierens und Anerkennens zu untersuchen, ggf. beginnend mit Zeige- und Aufforderungsspielen.

Tadel (als negative Sanktion) und Lob (als positive Sanktion) sind dabei selbst schon Teile einer gemeinsamen, wenn auch je auf die Teilnehmer aufgeteilten, Kontrolle des richtigen Verhaltens im Blick auf das gemeinsame Spiel. Sie spielen eine zentrale Rolle für den Erwerb der Fähigkeit, an gemeinsamen Verhaltensspielen teilzunehmen. Denn hier geht es ja darum, gewissermaßen prä-intentional an einem gerade laufenden gemeinsamen Spiel richtig teilzunehmen und Unrichtigkeiten je präsentisch zu bemerken. Die volle Intention, gerade dieses Spiel jetzt oder nachher in dieser oder jener Rolle spielen zu wollen, setzt dagegen schon eine symbolische, propositionale, Repräsentation des Spiels (etwa seine Benennung) bzw. der entsprechenden Teilrollen und Teilregeln voraus. Das heißt, ich kann auf überpräsentische Weise erst dann die Absicht haben, ein bestimmtes Spiel zu spielen sagen wir, Schach, wenn ich ein Wort für Schach schon kenne und weiß, wie das Wort dem Spiel zugeordnet ist. Erst dann kann ich das Schachspielen ,nach Belieben' aktualisieren oder auch nicht. Denn wenn mir (sagen wir zufällig) das Wort „Schach“ einfällt, brauche ich noch nicht automatisch das Schachbrett zu holen. Ich kann das tun oder auch lassen. Anders liegt der Fall, wenn das Kind zufällig einen Ball präsentisch sieht und mich einfach zum Spielen einlädt. In ähnlicher Weise interagieren auch schon Hunde mit ihren Herrchen.

Im Verlauf des Erwerbs von Spielkompetenzen ist das Lob häufig eine Art Default-Fall: Erwachsene loben schon, bevor alles richtig gekonnt ist. Das Lob sagt: Du gehörst zu uns. Du bist schon in die Gemeinschaft aufgenommen. Du nimmst an den gemeinsamen Spielen teil. In gewissem Sinn bedeutet ein solches Loben die Vergabe von Intentionalitätsvorschusslorbeeren (Henrike Moll). Allerdings hat diese Praxis der Behand- 
lung eines Wesens als potentielle Person, schon bevor diese ihre personalen Kompetenzen voll entwickelt hat, nur beim Menschen, nicht bei Tieren, den bekannten durchschlagenden Erfolg. Nur Menschen lernen die zentralen gemeinsamen Spiele, samt der Kontrollspiele des Tadelns und des Lobes. Das ist in gewissem Sinn einfach eine Tatsache, die man, wenn man unbedingt will, eine biologische Tatsache nennen kann. Sie ist als solche nicht weiter zu erklären, sondern einfach anzuerkennen.

$\mathrm{Ob}$ es Tiere gibt, die unter sich hinreichend analoge Spiele spielen, wissen wir faktisch nicht. Es ist aber keineswegs so, dass wir das grundsätzlich nicht wissen können, so wie wir grundsätzlich nie wissen können, wie genau es sich anfühlt, eine Fledermaus zu sein, solange wir nicht geklärt haben, was wir hier ,wissen“ und ,sich anfühlen“ zu nennen belieben. Die gestellte Frage ist nämlich so gemeint, oder sollte so verstanden werden: Gibt es hinreichend Anhaltspunkte dafür, dass, sagen wir, Delfine einander etwas zeigen können? Oder wären entsprechende Aussagen als unsere Projektionen in ihr Verhalten zu deuten, bisher jedenfalls?

Dabei kann es höchst interessant sein, genauer zu untersuchen, was denn die lerntheoretischen und lernpraktischen Differenzen sind, die sich ,empirisch'bzw. faktisch in wiederholbaren Beobachtungen und beliebig wiederholbaren Experimenten dingfest machen lassen. Solche Untersuchungen könnten zum Beispiel die Frage betreffen, welche Folgen ein (oft auch bloß subjektiver) Mangel an Lob und eine allzu stark auf Tadel und Kritik abgestellte Erziehung für die Entwicklung autonomer Personen hat. $^{11}$

\subsection{Voraussetzungen intentionaler Handlungen}

Personale Grundkompetenzen hängen von einer Kultur-Tradition ab. Das ist keine empirische Aussage. Es ist vielmehr eine Art Grundtatsache, die zu einer materialbegrifflichen Erläuterung von personalen Kompetenzen im Unterschied zu rein natürlichen (angeborenen) Fähigkeiten gehört. Diese Tatsache bedeutet nicht, dass wir Traditionen nie verändern, Rollenspiele nicht auch verweigern könnten. Wir können neue Institutionen vorschlagen und entwickeln, und zwar als schon gebildete Personen. Aber alle diese Fortentwicklungen finden längst schon im Rahmen einer Gemeinschaft und Tradition statt, in welcher bestimmt ist, was es heißt, als Person zu

\footnotetext{
${ }^{11}$ Es könnten zum Beispiel Personen, die eher durch Tadel als durch Lob das Richtige zu tun lernen oder gar durch Abrichtung und Zwang, konservativer sein als andere, also stärker übliche Traditionen fortführen als neue beginnen können.
} 
handeln, als Person einen Vorschlag oder auch nur eine Aussage, eine Proposition, zu machen und für diese zu argumentieren bzw. gegenüber anderen Personen zu begründen.

Insofern Handlungen begrifflich bestimmt sind durch Erfolgs- oder Erfüllungskriterien bzw. in der Praxis der Beurteilung, ob entsprechende (möglicherweise partiell explizit artikulierte) Handlungsversuche geglückt sind oder nicht, setzt jede personale Handlungskompetenz die Ebene der Gemeinschaft bzw. einer Gemeinschaftlichkeit der Beurteilung der Erfolge voraus. Und nicht nur das. Handlungsschemata werden als solche lehrend und lernend tradiert. Das heißt, wir lernen (in Abhängigkeit gewisser Ceteris-Paribus-Bedingungen, in welchen unter anderem situative Möglichkeitsbedingungen bestimmt sind) vorgegebene, generische" Handlungen , willkürlich ${ }^{\star}$ oder , auf Abruf ${ }^{\star}$ zu aktualisieren. Erst wenn ein Kind auf Aufforderung oder ,willkürlich' (etwa auch auf eine Art ,Selbstaufforderung' hin), sagen wir, den Tisch decken kann, beherrscht es die Handlung des Tischdeckens. Mitvorausgesetzt ist die - oft schon vor der eigenen Beherrschung der Handlung verfügbare - Fähigkeit der (mehr oder minder kompetenten) Teilnahme an der Beurteilung der Erfüllungsbedingung der Handlung. Dazu muss man ,wissen', wann der Tisch (vollständig) gedeckt ist, d. h. als gedeckt gilt, so dass die Handlung abgeschlossen, ihr Ende und Ziel erreicht hat.

Sprechen wir von Handlungen, welche wir ausführen, so unterscheiden wir schon zwischen den Einzelhandlungen, dem Handeln qua Tun, und den diesem konkreten Tun zugeordneten generischen Handlungen. Insbesondere dann, wenn die generischen Handlungen beliebig ausführbare Handlungen sind, sprechen wir auch von „Handlungsschemata“12. Als Beispiele denke man etwa an das Pfeifen einer Melodie oder gerade auch an das Rezitieren eines Textes, der dann immer auch komplexe Handlungsbeschreibungen enthalten kann.

Generische Handlungen bzw. Handlungsschemata sind keineswegs einfach Klassen von Einzelhandlungen oder Einzelakten, obwohl dies der Vergleich mit der Unterscheidung zwischen Einzelnem und Allgemeinem (Generischem) leider oft nahe legt. Besser ist schon der im Ausdruck „Handlungsschema“ mittransportierte Vergleich generischer Handlungen mit den Schemata einer Praxis wie der des Rechnens, in welcher beliebig richtig (aber auch falsch) ausführbare Regeln die Ausführung einer Rech-

${ }^{12}$ In ihrer Logischen Propädeutik (Mannheim 1967) sprechen Wilhelm Kamlah und Paul Lorenzen in Wiederaufnahme einer schon bei Kant angelegten Redeweise von (aktualisierbaren) Handlungsschemata. 
nung bestimmen. Dieser Vergleich möge aber seinerseits nicht vorschnell dahingehend ausgedeutet werden, dass alle Handlungsschemata schematisch befolgbare Verfahren wären. Vielmehr besteht jedes Handeln aus einer Art Mischung von beliebig Aktualisierbarem und widerfahrnisartigen Erfolgen und Misserfolgen, wobei die letzteren häufig weitere Korrekturhandlungen nötig machen. Kurz, wir wissen, dass in unserem Handeln immer auch etwas etwa ,durch Zufall' schief gehen kann. Daraus folgt aber nicht, das wir nicht (frei) handeln könnten, sondern nur, dass man dies nicht dem einzelnen glückenden oder missglückenden Akt unmittelbar entnehmen kann. Wir sprechen vielmehr in der Regel erst dann davon, dass einer eine Handlung X ausführt, wenn er in der Lage ist, $X$ in entsprechenden Situationen beliebig auszuführen. Das muss einer, der von sich ein Können (etwa des Tanzens) behauptet, uns gegebenenfalls zeigen, und zwar nach dem berühmten Spruch: Hic Rhodus, hic salta, hier ist Rhodus, hier musst du dein Können beweisen. Nur dann können wir ein Tun als Aktualisierung eines Handlungsschemas X verstehen, wenn wir das Können generisch durch Vorführungen zeigen können und nicht schon dann, wenn wir es bloß verbal uns oder anderen zuschreiben.

Sprechen und dann auch Denken können nun ihrerseits als Handlungsversuche glücken oder missglücken. Gerade dies zeigt, dass sie die kompetente Teilnahme an Handlungsformen voraussetzen. Als Personen müssen wir also immer schon ein gemeinschaftliches ,Spiel' konzeptueller, begrifflicher, wenigstens in Umrissen sprachlich artikulierter, Unterscheidungen beherrschen und eine gewisse Kompetenz der Bewertung von Erfüllungen gewisser Bedingungen besitzen.

Der Mensch ist ein sprachbegabtes Wesen. Aber nur als Personen beherrschen wir Begriffe, im Unterschied zur bloßen Beherrschung der Artikulationsformen des Sprechens. Letztere können auch Papageien antrainiert werden. Eine Person zu sein, die Begriffe beherrscht, bedeutet, die gemeinsamen Normen zu kennen, die sich aus dem Begriffsgebrauch in Sprechakten ergeben.

Unsere Möglichkeiten der Entwicklung personaler Kompetenzen hängen dabei wesentlich ab von den realen Gemeinschaften, besonders auch von der Primärgemeinschaft wie der Familie, in deren Tradition das Individuum lebt und sich als Person entwickelt. ${ }^{13}$

${ }^{13}$ So dürfte z.B. kaum bezweifelt werden, dass die (frühe) Entwicklung Mozarts ohne die erzieherischer Begabung (und die enorme Autorität) seines Vaters nicht möglich gewesen wäre. Aber auch unsere gesamte technisch-wissenschaftliche und politische Weltverfassung verdankt vieles den Kultur-Leistungen der Antike. Es gibt dann auch 
Die Praxis nun, welche Bewusstsein und Selbstbewusstsein bzw. das zugehörige Denken und Selbst-Denken sowohl möglich macht, als auch verlangt, ist selbst schon eine Praxis der interpersonalen Beziehung zu anderen Personen. Erst in zweiter Linie, in einem logisch gestuften Aufstieg, wird daraus eine Praxis intrapersonaler Selbstbeziehungen.

Es handelt sich im Fall der interpersonalen Beziehungen zwischen Personen, ganz grob gesagt, um sprachlich, wenigstens symbolisch, gestützte Kommunikations- und Kooperationsbeziehungen. Konkreter sind es Sprechhandlungen verschiedenster Art, unter denen besonders die Sprechhandlungen der Aussage, des Versprechens bzw. schon der schwächeren Selbstverpflichtung in der Absichtserklärung und dann auch der Begründung zentrale Prototypen für weitere, vielfältig ausdifferenzierbare, Sprechhandlungen sind. Intrapersonale Beziehungen der hier relevanten Art kennen wir aus unseren stillen verbalen Planungen des Überlegens. Sie setzen schon voraus, dass wir entsprechende interpersonale Rollen spielen können. Daher bin ich Person erst dann, wenn ich die relevanten zwischenpersonalen Verhaltens- und Handlungsformen beherrsche. Damit wird der Kompetenzbegriff der Person zu einem nur kultürlich zu begreifenden Rollen- und damit Relationsbegriff. Eine Person zu sein ist also keine natürliche Eigenschaft des Individuums. Person im Kompetenzsinn des Wortes (neben dem es aber auch noch einen moralischen Sinn, den Würde- und Wertungsbegriff des Menschen oder der Person gibt) bin ich in dem Ausmaß, in dem ich zur Klasse der Menschen gehöre, welche in der Lage sind, an einer gewissen Form gemeinsamen Lebens teilzunehmen.

\subsection{Ich-Modus und Wir-Modus}

Das Tier lebt nur im Ich-Modus. Das heißt, es nimmt einfach nicht teil an einer Praxis gemeinsamen Wissens, samt der dafür begrifflich notwendigen gemeinsamen Richtigkeitskontrollen, und zwar weder mit uns noch, nach allem unserem Wissen, mit anderen Tieren. Insofern, aber auch nur insofern, ähnelt das Tier den Maschinen, nämlich im Hinblick auf einen Mangel an selbständiger Kontrolle des Richtigen. Es unterscheidet sich von Maschinen und auch von Pflanzen wesentlich aufgrund seiner Subjektivität. Diese Subjektivität besteht in einer inneren und äußeren Sensitivi-

so etwas wie Grade in der Entwicklung der Person, und zwar wenn man an den Umfang ihrer Handlungskompetenz denkt. Erst recht gibt es Grade der Entwicklung der Persönlichkeit, wenn man unter diesem Titel die (vernünftige) Urteils- und autonome Regelsetzungskompetenz einer Person zusammenfasst. 
tät, der Fähigkeit zur Empfindung von Schmerz, Begierde, Befriedigung und Frustration, und einer gewissen Fähigkeit der sinnlichen Wahrnehmung präsentischer Umwelt, samt differentieller Antworten im Tun, also in der Selbstbewegung des Tieres.

Wir sollten nun entsprechend unterscheiden zwischen dem animalischen Bewusstsein als der präsentischen awareness in Bezug auf innere Empfindungen (sensations oder inner experiences) bzw. attention auf präsentisch wahrnehmbare Objekte auf der einen Seite, dem menschlichen Bewusstsein oder der conscientia im Sinne einer Teilnahme an einer gemeinsamen Kontrollpraxis des rechten Urteilens, Schließens und Handelns andererseits. Dann nämlich begreifen wir auch, in welchem Sinn Tiere kein Bewusstsein haben, und zwar nach all unserem Wissen. Dass Tiere kein menschliches Bewusstsein haben, das ist reine Tautologie. Dass sie kein Bewusstsein im Sinn der conscientia, des gemeinsamen Wissens über das, was als richtig oder unrichtig, wahr oder falsch zu werten ist, dass ist keineswegs tautologisch. Tieren und Menschen gemeinsam ist dagegen eine subjektive Sensitivität der inneren Empfindung und der nach außen gerichteten Vigilanz und attentiven Aufmerksamkeit der sinnlichen Wahrnehmung, samt der dadurch mögliche Selbststeuerung der Bewegung des Körpers im animalischen, begierdegesteuerten, Verhalten. Nicht gemeinsam ist die conscientia, die gemeinsame und dabei immer auch eigene Kontrolle des Richtigen, das aus einer transpersonalen eben damit auch partiell transsituativen Perspektive, eben im Modus des Wir, bestimmt ist.

Insbesondere ist die menschliche Wahrnehmung verschieden von der Wahrnehmung eines Tieres. Auch das ist zunächst eine Tautologie. Gemeint ist aber, wie John McDowell seit Erscheinen seines äußerst wichtigen Buches Mind and World nicht müde wird zu betonen, dass der Inhalt menschlicher Wahrnehmung, als das Wahrgenommene, längst schon begrifflich gefasst ist, dass also der Inhalt der Wahrnehmung und der Inhalt eines passenden Wahrnehmungsurteils, mit dem man den Akt des Wahrnehmens möglicherweise begleiten könnte, als Inhalt identisch sind. Das ist der Grund warum Wahrnehmungen prämissenlose Ausgangpunkte im schließenden Denken sein können und warum in Wahrnehmungen Begründungsketten enden können, warum also Wahrnehmungen (manchmal sogar ausschlaggebende) Gründe dafür sein können, dass wir (oder jemand) etwas weiß bzw. dass etwas der Fall und eine entsprechende Aussage wahr ist. Mit anderen Worten, das Wahrnehmen eines Tieres unterscheidet sich kategorial von menschlicher Wahrnehmung. 
Ein richtiges und falsches deiktisches und symbolisches Benehmen und dann auch ein richtiges und falsches symbolisches (Sprech-)Handeln gibt es nun aber selbst immer nur auf der Grundlage eines möglichen gemeinsamen Wissens, der conscientia. Allerdings ist es etwas anders, das Benehmen direkt und präsentisch als richtig zu kontrollieren und eine RePräsentation von nicht Präsentischem als wahr zu beurteilen. In der Tat macht erst eine gemeinsame Praxis des symbolischen Handelns richtige oder falsche symbolische Re-Präsentationen von nicht präsentisch zu- oder vorhandenen Gegenständen oder Ereignissen möglich, oder dann auch von bloßen Möglichkeiten. Das geschieht über eine am Ende immer präsentische Überprüfung der Zuordnungsbedingungen zwischen Aussage und Wahrnehmung, die (der Idee nach) zu einem allgemein anerkennbaren Urteil über deren Erfülltheit oder Richtigkeit führt.

Es ist bedeutsam, dass die präsentische Ausführung der für den Menschen wichtigsten symbolischen Handlungen, der Sprechhandlungen in der Lautsprache, für sich betrachtet praktische keinerlei Aufwand an Kraft und Zeit erfordern, sondern, wenn die Handlungformen erlernt sind, spontan ausführbar sind. Das heißt, wenn uns ein Wort spontan, insofern passiv, einfällt, können wir es sofort, spontan, insofern aktiv äußern, etwa im Unterschied dazu, dass ich ein Bild erst malen muss und dazu die erforderlichen Zusatzmittel brauche. Insofern können (und sollten) wir zwischen der Spontaneität eines symbolischen Sprachhandeln und der Willkürfreiheit anderer Handlungen, die ich ebenfalls nach Belieben ausführen kann, sagen wir dem Fahrradfahren, unterscheiden: Ich kann jetzt, um 12.00, spontan den Wunsch oder die Absicht fassen oder das Versprechen artikulieren, um 14.00 Fahrrad zu fahren. Und ich kann, wenn ich will, diese Wunschabsicht oder dieses Versprechen um 14.00 nach Belieben ausführen, wenn das Fahrrad zuhanden ist und mich nichts anderes von der Ausführung abhält. Dabei sind dann allerdings, und das ist für jede begrifflich angemessene, nicht mythologisch-dogmatische, Handlungsanalyse absolut zentral, andere Wunschabsichten keine Ursachen der Verhinderung, sondern bestenfalls mögliche Gründe für eine Änderung der handlungsleitenden Absicht. Die handlungsleitende Absicht wird im Handeln selbst wirksam, und zwar auch dann, wenn der Ausführungsversuch aufgrund unvorhergesehener Ereignisse, insofern zufällig, scheitern sollte. Diese Wirksamkeit ist nun eine kausale Beziehung nur im englischen, fast uferlosen, Gebrauch des Wortes „,causal“. In Wirklichkeit ist nämlich schon die Beziehung zwischen dem spontanen Einfall und dem Fassen einer Absicht in einer freien Planentscheidung nicht kausal, schon gar nicht die zwischen der Entschei- 
dung und ihrer willentlichen Ausführung, jedenfalls nachdem ich die Handlung nach Belieben auszuführen oder zu unterlassen gelernt habe. Der Grund für diese Verweigerung des Wortes „kausal“ liegt daran, dass die genannten Beziehungen von ganz anderer Art sind als jede causa efficiens der Physik. Und das wissen wir aus unserer praktischen Erfahrung im Handeln auch sehr gut, so gut, dass dieses Wissen jeder experimentellen Wissenschaft längst schon zugrunde liegt und daher von dieser auch nie widerlegt, bestenfalls in Einzelfällen präzisiert, werden kann. Was uns hier irreführt, sind unsere Formen des Redens und die notorischen Mehrdeutigkeiten unserer Wörter. Ohne eine Differenzierung zwischen Gründen und Ursachen im Handeln, ferner zwischen den prima facie bloß symbolisch artikulierten Wunschabsichten (in deren expressiven Anerkennung das Fassen der Absicht besteht) und den erst im Tun wirklich wirksam werdenden handlungsleitenden Absichten im perfektiven Sinn wird man das menschliche Handeln begrifflich nie angemessen zu fassen bekommen.

Wegen des Mangels an Normen des Richtigen gibt es beim Tier kein Denken und kein Sprechen bzw. kein symbolisches Handeln. Wer Tieren dennoch ein Denken oder einen Symbolgebrauch zuspricht oder zuschreibt, wie inzwischen sogar viele Enzyklopädien und Wörterbücher, der gebraucht einen im Hinblick auf den traditionellen Kerngebrauch viel zu weiten, metaphorischen, Begriff des Denkens bzw. des symbolischen Handelns. Dabei verführt die ambivalente Rede von einer ,Zeichenpraxis“ leicht dazu, die kategoriale Differenz zwischen einer normativen Handlungspraxis im Wir-Modus und eines bloßen kollektiven Verhaltens jeweils im Ich-Modus zu übersehen.

Reden und Handeln, wie wir es verstehen, ist also nur in einer Gemeinschaft von Handelnden und Sprechenden, im Wir-Modus und unter der Leitung eines allgemeinen Mit-Wissens, also eines Bewusstseins als allgemein verfügbarer Kontrollmöglichkeit möglich. Eben deswegen ist sie nur den Menschen möglich. Es ist eben daher eine szientistische Verengung, wenn man Handeln und Sprechen als bloßes Verhalten im IchModus behavioristisch beschreibt und durch gewisse Verhaltensdispositionen oder dann auch durch ein System von Operationsregeln etwa des Gehirns kausal erklären will. Die Annahme, ein womöglich im Gehirn repräsentiertes Regelsystem oder Verhaltensschema im Sinne eines dispositionellen Reaktionsschemas bestimmte das individuelle Tun, ist dagegen schon rein spekulativ, ohne jede Erfahrungsstütze.

Jedes Handeln ist dabei Ausführung von vielen verschiedenen Handlungschemata zugleich. Indem ich jetzt etwa diesen Text schreibe, tue ich 
vielerlei: Ich überlege, spreche still mit mir selbst, bewege Hände und Finger, übersetze (stille) Rede in geschriebene Sprache usf. Ich tue dabei also alles das, von dem ich, auf Nachfrage, sinnvollerweise sagen kann, dass ich es tue. Dabei sind Frage und Antwort immer mit einer bestimmten Betrachtungsweise meines Tuns verbunden: Antworten auf Fragen nach dem Ziel und Sinn meines Tuns unterscheiden sich von Antworten auf Fragen nach dem besonderen Weg, den ich dabei einschlage oder auf Fragen nach einer angemessenen Oberflächenbeschreibung meines Tuns, das dann schon eher in der Perspektive des Verhaltens erscheint.

Wenn wir zwar von einem Akteur A sagen können, er verhalte sich gemäß dem Schema X, wenn A also, deskriptiv bzw. askriptiv, also im Modus einer äußeren Zuschreibung gesprochen, $\mathrm{X}$ zwar tut, er aber $\mathrm{X}$ gar nicht tun will oder ihm vielleicht (vor oder) während seines Tuns gar nicht bewusst ist, dass er $\mathrm{X}$ ausführt, sei es, dass er überhaupt nicht weiß (sagen kann), was er tut, sei es, dass er glaubt (sagen würde), etwas anderes als $\mathrm{X}$ zu tun, dann wollen wir $\mathrm{X}$ bloß das Verhaltensschema seines Tuns nennen. Ein Tun ist eine bloße Verhaltung, wenn es vom Akteur nicht bewusst unter ein Handlungsschema gestellt ist.

Wenn einer z.B. gähnt, mag dies eine Verhaltung sein. Hält er dabei die Hand vor den Mund, so mag sich dies so automatisch ergeben, dass er es gar nicht mehr eigens zu wollen braucht. Er wird in der Regel zwar wissen (sagen können), was er tut, aber er wird sich zu diesem Tun nicht etwa entschließen müssen. Natürlich kann einer auch absichtlich sein Gähnen zu Schau stellen, etwa um zum Ausdruck zu bringen, dass er sich langweilt.

Das Tun von Tieren besteht in der Regel aus bloßen Verhaltungen im ich-Modus. Wir sagen zwar, dass Tiere dieses oder jenes Schema ausführen, dass sie sehen und hören, sich untereinander und mit Menschen in gewissen Dingen verständigen können. Askriptiv sagen wir sogar, Tiere hätten Absichten, etwa wenn wir sagen, die Katze wolle mit der gefangenen Maus erst einmal spielen. Und wir sagen manchmal auch, rein askriptiv und eben damit metaphorisch, dass ein Tier überlege oder sich zu etwas entschließe. Wir nehmen dabei jedoch nicht wirklich an, dass Tiere in dem Sinne etwas wollen können, dass sie ihre weiteren Verhaltungen selbst unter einen Handlungsplan A statt etwa ein alternatives Handlungsschema B stellen, Handlungsabläufe also sprachlich repräsentieren und das Tun als Erfüllung oder Nichterfüllung entsprechender (sprachlich artikulierbarer) Bedingungen kontrollieren können: Alle unsere Erfahrungen zeigen, dass 
sie dies jedenfalls für etwas komplexere, auf der Basis vergangener Erfahrungen in die Zukunft weisende Verhaltensschemata nicht können. ${ }^{14}$

\subsection{Autonomie als bewusste Antwort auf Traditionen und Institutionen}

Auf höherer Ebene ist es dann interessant zu prüfen, wann, erstens, eine ,Abweichung' von einem bisher gespielten Spiel als Fehler erkannt bzw. , sanktioniert' wird, wann dagegen ein ,neuer Zug' im Spiel als eine Art neuer Regelungsvorschlag bzw. als Teil eines ,neuen' Spiels anerkannt wird, mit neuen ,Normen' oder ,Richtigkeiten'. Diese Normen oder Richtigkeiten existieren in der Form gemeinsamer Anerkennung, dass noch ,richtig' mitgespielt wird, also in der Abwesenheit eines entsprechenden Protest- oder Sanktionsverhalten. Drittens gibt es die Möglichkeit, dass eine Abweichung als Teasing oder Witz verstanden wird.

Institutionen und Traditionen sind dabei als ,normative' Praxen der $\mathrm{Zu}$ schreibung von Pflichten oder Erwartungen, von Erlaubnissen oder Verboten bestimmt, und durch die Kontrolle ihrer Erfüllungen. Vorausgesetzt ist die - zunächst implizite oder empraktische - Anerkennung der Zuschreibungen durch handelnde Personen.

Erfüllungen werden kontrolliert in Bewertungs-Spielen der lobenden Anerkennung und tadelnden Kritik. In diesem Spiel sind wir alle zugleich immer auch ,Schiedsrichter', und urteilen dabei in der Rolle des Beobachters. Als Schiedsrichter oder , score-keeper ' beurteilen wir das Tun von anderen und auch von uns selbst im Blick auf allerlei Erfüllungs- oder Erfolgsbedingungen. Diese können durch implizite Sitte und Tradition gegeben sein. Sie können sich aber auch in expliziten Zweck- und Erfolgsbewertungen niederschlagen. Wir können etwa frei vorschlagen, dass es gut ist, so zu urteilen, und dass andere uns daher zustimmen mögen. Dies können wir unabhängig davon, ob es schon implizit anerkannte Normen oder explizit formulierte Prinzipien oder Regeln gibt, die ohne weiteres bestimmen würden, was es in diesem Fall heißt, richtig zu urteilen. Dieser Fall, den wir von ästhetischen Urteilen her kennen und den Kant als erster analysiert, ist, wie Hegel und Wittgenstein sehen, viel häufiger, als wir glauben. Ob er so häufig ist, wie Rorty glaubt und Brandom zu glauben scheint, steht auf einem anderen Blatt.

${ }^{14}$ Dass sich der Mensch vom Tier gerade durch seine Sprachfähigkeit und sein (Sprach)Gedächtnis unterscheidet, wie dies Platon und Aristoteles, Descartes und Hobbes, Nietzsche und viele andere längst schon sahen, bestätigt sich hier erneut. 
Wenn wir nun so die hinreichende oder unzureichende Erfüllung bzw. die Nichterfüllung von ,Kriterien' beurteilen oder im freien Urteil paradigmatisch neue Urteilsformen vorschlagen und zu ihrer Anerkennung gewissermaßen aufrufen, dann sind wir selbst keineswegs ,bloße' Beobachter. Denn erstens ist jedes derartige Urteil auch eine Bewertung, wie schon die Bewertung der Richtigkeit der Befolgung einer Regel oder Handlungsform, etwa auch beim sprachlichen Handeln, immer zugleich auf schon anerkannte Wertmaßstäbe oder Normen gemeinsamen Handelns verweist, bzw. diese Normen vorschlagsartig, weiterentwickelt‘. Und zweitens können wir unsererseits immer auch von anderen daraufhin beurteilt werden, ob wir ,gute' Schiedsrichter sind, also dieses Spiel hinreichend gut spielen oder nicht. Es gibt im Schiedsrichterspiel keinen Oberschiedsrichter. Es gibt auch selten oder nie ,letzte Kriterien" der Richtigkeit einer Regelbefolgung, auch wenn im Bereich schematischer und mathematischer Regeln wenig offen scheint, sofern man den Umgang mit ihnen beherrscht. Diesen Umgang wiederum kann im Prinzip jeder lernen und kontrollieren. Wichtig ist dabei, und eben das hat uns Wittgenstein zu zeigen versucht, dass die praktisch befriedigende Anerkennung in einer humanen Gemeinschaft über die Korrektheit eines jeden Versuchs einer Regelbefolgung entscheidet. Die Identität einer jeden Regel selbst und, allgemeiner, die Identität irgend einer Bedeutung, eines Sinnes und dann auch irgend eines repräsentierbaren, benenn- und beredbaren Gegenstandes ist daher, wie vor Wittgenstein schon Hegel sieht, nicht außerhalb der Form und Idee dieses gemeinsamen Urteilens bestimmt.

\section{LITERATUR}

Anscombe, G.E.M. 1957 Intention, Oxford: Oxford Univ. Pr.; dt.: Absicht. Freiburg u. München 1963 bzw. 1986.

Bennett, J.F. 1976 Linguistic Behaviour, Cambridge Univ. Pr.; dt.: Sprachverhalten. Frankfurt 1982.

Brandom, R.B. 1994 Making It Explicit. Reasoning, Representing and Discursive Commitment, Cambridge/Mass.: Harvard Univ. Pr. 1994, dt.: Expressive Vernunft. Frankfurt/M. 2000.

- 2000 Articulating Reason. An Introduction to Inferentialism, Cambridge/Mass.: Harvard Univ. Pr. 2000, dt.: Begründen und Begreifen. Eine Einführung in den Inferentialismus. Frankfurt/M. 2001.

Carnap, R. 1928 Scheinprobleme der Philosophie, Berlin. Neudruck mit 1928a: Hamburg 1961.

Chomsky, N. 1980 Rules and Representations, New York: Columbia Univ. Pr., 1980. 
- 1973 Reflexions on Language, New York; dt. Reflexionen über die Sprache. Frankfurt/M. 1977: Suhrkamp.

Davidson, D. 1980 Essays on Actions and Events, Oxford: Oxford Univ. Pr., dt.: Handlung und Ereignis. Frankfurt/M.: Suhrkamp 1990.

- 1984 Inquiries into Truth and Interpretation, Oxford: UP.

Kambartel, F. 1977/78 „Symbolic Acts“, in G. Ryle (ed.), Contemporary Aspects of Philosophy, Stocksfield 1977, 70-85; dt. „Symbolische Handlungen“; in: J. Mittelstraß / M. Riedel (eds.), Vernünftiges Denken, Berlin: de Gruyter 1978.

Kern, A. 2006 Quellen des Wissens. Zum Begriff vernünftiger Erkenntnisfähigkeit, Frankfurt (Suhrkamp) 2006.

Krämer S. / König E. 2002 Gibt es eine Sprache hinter dem Sprechen?, Frankfurt/ M.: Suhrkamp.

McDowell, J. 1994 Mind and World, Cambridge/Mass.: Harvard Univ. Pr. und London 1994.

Quine, W.V.O. 1960 Word and Object, Cambridge/Mass: MIT Pr. 1960 dt.: Wort und Gegenstand. Stuttgart: Reclam.

Rödl, S. 1998 Selbstbezug und Normativität, Paderborn: Schöningh.

Rorty, R. 1991 Objectivity, Relativism, and Truth. Philosophical Papers I, Cambridge etc.

von Hayek 1996 Die verhängnisvolle Anmaßung: Die Irrtümer des Sozialismus, Tübingen: Mohr/Siebeck 1996 (engl. 1988). 
